\title{
Study on Goal Directed Alternative Plan Recommendation Agent Negotiation
}

\author{
Botao Zhong Lieyun Ding Zhenwen Zhang \\ Inst. of Systems Engineering, Huazhong Univ. of Science and Technology, Wuhan 430074, P.R. China
}

\begin{abstract}
During the negotiation, many conflicts are due to the incomplete or incorrect knowledge about the negotiation goal. Negotiators can avoid it through asking the underling goals. We focus on the underlying goal of negotiation and present Goal Directed Alternative Plan Recommendation Negotiation (GDAPLN), in which a negotiation agent can ask for the underlying goals of negotiation. After getting the underlying goal, the negotiation agent argues over the goals 、 beliefs behind the goals and plans, and recommend alternative plans to achieve this underlying goal. If the alternative plan is more attractive, the respondent may change its initial plan and preference. We give the negotiation protocol named "AskMore" and present a conceptual model for GDAPLN and describe the functions of its components.
\end{abstract}

Keywords: Goal directed agent negotiation, Alternative plan recommendation, Consistency checking

\section{Introduction}

Goals are important based on the notion: any desire or preference that can lead to the generation and adoption of goals (which further affects the outcome of the reasoning or plan task) intends to satisfy those goals. The negotiation agent which focuses on negotiating by interests or goals rather than positions may increase the quality of agreement and the speed of reaching the agreement [1]. During the negotiation, many conflicts or failure are due to the incomplete or incorrect knowledge about the negotiation goal. Negotiators (proponent \& respondent) can avoid this kind conflict through asking the underling goals of negotiation. In this paper, we mainly focus on the underlying goal of negotiation and present Goal Directed Alternative Plan Recommendation Negotiation (GDAPLN), in which a negotiation agent can ask his respondent for the underlying goals of negotiation when the negotiation goes into stalemate. After getting the underlying goal of negotiation, the negotiation agent argues over the goals v the beliefs behind the goals and plans to achieve the goals, and recommend alternative plans to achieve this underlying goal. If the alternative plan is more attractive, the respondent may change his initial plan and preference and the negotiation process can avoid the conflicts other than solve the conflicts to make the negotiation successful. We give a conceptual model for GDAPLN and describe the functions of its components. Specially, consistency checking 、 conflict discovery 、 belief revisal and alternative plan recommendation is given.

The remainder of this paper is organized as follows. In Section 2, we give the protocol and the process for GDAPLN. In Section 3, a conceptual model for GDAPLN is proposed and the components of it depicted in detail. In Section 4, an example is given to illustrate our model and the negotiation process. Related researches and conclusion follow in Section 5, Section 6.

\section{Protocol for Goal Directed Altern -ative Plan Recommendation Agent Negotiation}

Proponent may form a more complete and accurate understanding of the respondent through asking the underling goals of negotiation. Sometimes, it is difficult or costly to resolve the conflict met during negotiation, but it may be easier to avoid the conflict and find alternative plans to reach the goal. This section describes the interactive protocol (Fig.1) for GDAPLN. The protocol is the extension of the classical alternating offer bargaining protocol. The extended part of the protocol, depicted in dashed line, called "AskMore", allows the proponent to request his respondent for the underlying goal and recommend alternative plans with possible refusal or acceptance at each stage. "AskMore" strategy is a complement for the classical alternative offer bargaining protocol. The proponent requests the respondent for the underlying goal of the negotiation. The respondent can inform the initiator of this or refuse to answer. In the case where the respondent informs the proponent of his underlying goal, the proponent can be clueless and quit the protocol with a cancel message, or he can argue over the underling goal with the respondent (During arguing, both negotiators can decide to accept the argument, 
provide counter-argument, ignore the argument, or terminate the entire conversation.), or he can recommend an alternative plan(s) to achieve the underlying goal to the respondent (under the hypothetic existence of alternative plan(s) to achieve the underlying goal). "AskMore”, as a strategy, rests on the hypothetic existence of alternative plan(s) to achieve the underlying goal of the respondent involving different resources for which bargaining will be more advantageous for both parties.

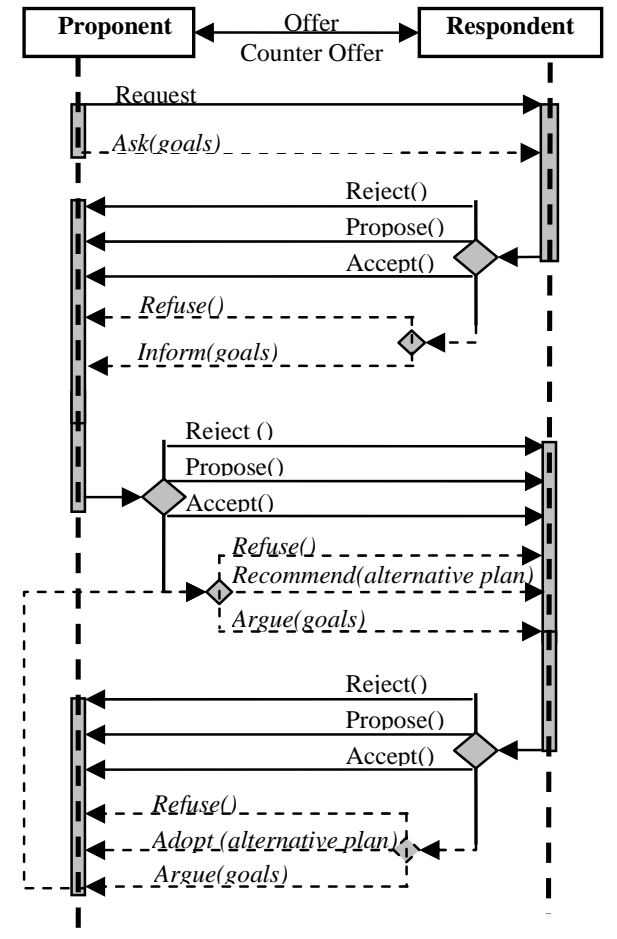

Fig.1: The protocol for GDAPLN.

\section{Conceptual model for GDAPLN agent}

In this section, a conceptual model for GDAPLN is designed according to the BDI Model. It includes belief base, goal base, plan base, reason engine and negotiation engine, and can be defined as: GDNA $=<B$, $G, P, S, R e, N e>$, where, $B$ is the Belief Base, $G$ is the Goal Base, $P$ is the Plan Base, $S$ is the Strategy Base, $R e$ is the Reason Engine, Ne is the Negotiation Engine.

Belief Base stands for the current beliefs of the agent. Typically, beliefs include facts about static properties of the negotiating application domain, and beliefs acquired when the agent executes its reasoning and negotiation. These will typically be conclusions about the current negotiation, and they can be inconsistent or even wrong and may change with the progress of the negotiation.

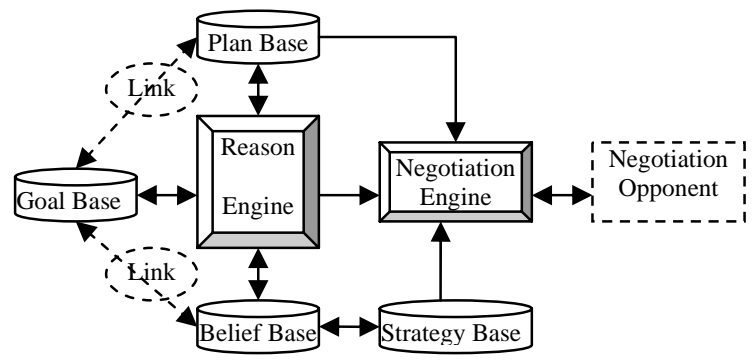

Fig.2: The conceptual model for GDAPLN agent.

Goal Base includes both intrinsic goals of the agent (its desires) and adopted goals of the agent (its intentions). This allows representation of a wide variety of goals, including goals for achieving maximum price, goals for shorter bargaining time and so on. The adopted goals are the current negotiation tasks, which will be chosen for execution either immediately or at some later time.

Plan Base holds the agent's current plans about how to achieve the adopted goal of the agent or react to certain bids from other agents. In the Plan Base, each plan is defined by a set of organized actions and (sub)plans to be executed towards the adopted goal. In more detail, a plan usually includes the negotiation issues, the negotiation sequence, namely the agenda, the reference and utility decision functions, attitudes to respondent and so on[3].

It can be seen from the conceptual model, the plans connect with the goals, and the goals connect with the beliefs. (1)The goals connect with the beliefs. For example, a person with the goal of purchasing a knowledge management soft might hold the belief that the soft can improve the sharing of the private knowledge and further improve the core competency power. A person with the goal of buying the hardware might base on a belief that they need the new hardware to increase productivity. The beliefs can be seen as the context in which the goal holds, and we can say that the beliefs justify the goal. If the context turns out to be unsatisfied, the agent would no longer have a reason to keep its goal. (2)The plans connect with the goals. Usually, there is more than one plan that can be executed to reach a given goal. However, because of incomplete or improper belief, different negotiator may select different plan with different preference to try to achieve the goal, sometimes plan is improper to achieve the goal. When meeting conflicts and difficulty in the negotiation, and reaching an agreement is getting difficult, proponent may try to persuade his respondent to change their minds by recommending alternative plans to avoid these conflicts other than try to resolve these conflicts, especially when these conflicts are very difficult to resolve and may cause the negotiation failure.

Strategy Base provides negotiation strategies and decision-making approaches by which participants 
attempt to achieve their goals. It determines which possible alternative action the agent will choose at each step. Each agent's strategy will strongly depend on the type of application it is involved in.

Reason Engine has two main functions: (1) Consistency Checking: allow the proponent to compare the respondent's justification with its own belief and find any conflicts between them. (2) Belief Revisal: with the exchange of information during the negotiation, the wrong belief about the respondent is revised and renewed, and this change will trig the reasoning process as seen in Fig.3. The negotiation is also the process for the negotiators to gain more complete information about the environment. (3) Alternative Plan Recommendation: to recommend alternative plans and provide justifications for the new alternative plans. If the alternative plan is more attractive to the respondent, the respondent may change the original plan, and negotiate about the newly recommended plan, trying to reach his underlying goals.

The whole reason process can be illustrated in Fig.3. During the negotiation process, Reason Engine treats the change of beliefs in the belief base as an event that trigs the following reason steps.

Firstly, through the Goal-Belief consistency checking, to check whether the current goal is consistent with the new belief or not, if not, reselect (or create) the new negotiation goal set from the Goal Base.

Secondly, through the Goal-Plan consistency checking to check whether the plan is consistent with the adopted goal or not, if not, select (or generate) new negotiation plans from the Plan Base. If there are more than one plans to achieve the adopted goal, select the most appropriate one as his current negotiation plan.

Finally, with the aid of the strategy base, instantiate the negotiation plan. Negotiation Engine executes this negotiation plan, if the execution fail, recommend the other alternative plan to continue negotiation, until the negotiation succeed or all alternative plans in the plan set are selected or the negotiation deadline reaches.

The consistency checking is one of core functions of the Reason Engine. In order to make the process automated, represent the belief base as a set of rules in Proposition Logic.

Let $B=\left\{p_{1} \rightarrow q_{1}, p_{2} \rightarrow q_{2}, \cdots, p_{n} \rightarrow q_{n}\right\}$, is the set of all Proposition Logic rules in Belief base; $p_{i} \rightarrow q_{i}$ is a rule, $p_{i}$ is the pre-conditions, $q_{i}$ is the conclusion, $p_{i}$ is the justification $q_{i}$, " $\neg$ " denotes negation.

$$
\begin{aligned}
V^{B} & =\left\{\bigcup_{i=1}^{n} p_{i}\right\} \cup\left\{\bigcup_{i=1}^{n} \neg p_{i}\right\} \cup\left\{\bigcup_{i=1}^{n} q_{i}\right\} \cup\left\{\bigcup_{i=1}^{n} \neg q_{i}\right\} \\
& =\left\{v_{1}, v_{2}, \cdots v_{m}\right\}
\end{aligned}
$$

is the atom set of $B / / B$ is consistent

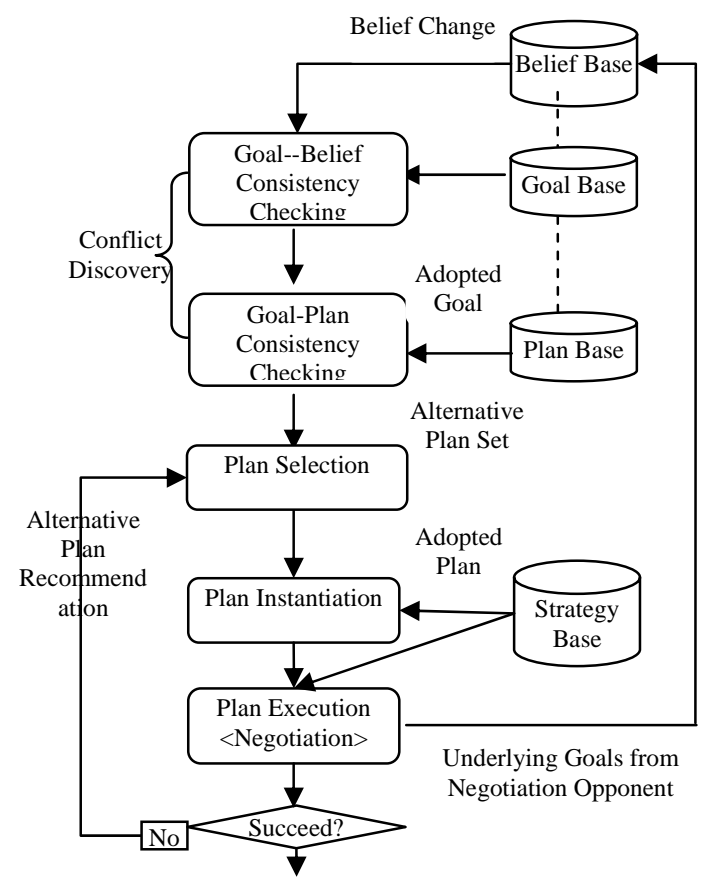

Fig.3: The reasoning process for alternative plan recommendation

$E^{B}=\left\{\left(\neg p_{i}, q_{i}\right),\left(\neg q_{i}, p_{i}\right) \mid\left(p_{i} \rightarrow q_{i}\right) \in B\right\}$

$G^{B}=\left(V^{B}, E^{B}\right)$ is defined as the graph representation of $B$

Let $m \times m$ matrix $A_{m \times m}^{B}$ is the matrix representation of $B$, which is the transitive closure of the adjacency matrix of $G^{B}$, where $A_{h \times m}^{B}\left[v_{i}, v_{j}\right]=\left\{\begin{array}{c}1, \text { if } v_{i}=v_{j} \\ \quad \text { or exists a directed path from } v_{i} \text { to } v_{j} . \\ 0 \text { otherwise }\end{array}\right.$ $A_{m \times m}^{B}$ can be got with using Warshall Algorithm.

A number of different approaches can be used to design conflict detection ranging from a simple arbitration heuristic to a more complicated de-feasible system that is based on the strength of justification or even a repeated learning heuristic [2]. Since the belief base is presented in Proposition Logic rule, the algorithm for consistency checking is given as follows.

Consistency Checking Process:

Elicit the rules from the justification, when getting the underlying goals and its justification from the respondent.

Let $R_{j}^{r}=\left(p_{j}^{r} \rightarrow q_{j}^{r}\right)$ is a rule from the respondent's $j t h$ justification; $B \cup R_{j}^{r}$ is the set of rule, which contains 
the proponent's belief and the respondent's jth justification $R_{j}^{r}$.

(1) Let $V^{B \cup R_{j}^{r}}=\left\{v_{1}, v_{2}, \cdots v_{m}\right\}$ is the atom set of $B \cup R_{j}^{r}$, Construct a directed graph $G^{B \cup R_{j}^{r}}=\left(V^{B \cup R_{j}^{r}}, E^{B \cup R_{j}^{r}}\right)$, corresponding to $B \cup R_{j}^{r}$.

(2)Find whether there are directed paths from $v_{i}$ to $\neg V_{i}$ and from $\neg V_{i}$ to $v_{i}$ in $B \cup R_{j}^{r}$.

$<1>$ if $\nexists v_{i} \in V^{\left(i+r_{j}\right)}$ satisfy $v_{i} \rightarrow\left(\neg v_{i}\right)$ or $\left(\neg v_{i}\right) \rightarrow v_{i}$, which indicates no conflicts exist between each other or need more additional knowledge.

$<2>$ if $\exists v_{i} \in V^{\left(i+r_{j}\right)}$ satisfy $v_{i} \rightarrow\left(\neg v_{i}\right)$ or $\left(\neg v_{i}\right) \rightarrow v_{i}$, which indicates conflicts exist between each other. The proponent sends $v_{i} \rightarrow\left(\neg v_{i}\right)$ or $\left(\neg v_{i}\right) \rightarrow v_{i}$ to the respondent, as the proof against the respondent's justification. At the same time, recommend the alternative plan to the respondent (the recommendation process and algorithm discussed in following section).

Alternative Plan Recommendation Process:

Let $B=\left\{p_{1} \rightarrow q_{1}, p_{2} \rightarrow q_{2}, \cdots, p_{n} \rightarrow q_{n}\right\}=\left\{\bigcup_{i=1}^{n} p_{i}\right\} \cup\left\{\bigcup_{i=1}^{n} q_{i}\right\}$

// $B$ is consistent.

Let $V^{B}=\left\{\bigcup_{i=1}^{n} p_{i}\right\} \cup\left\{\bigcup_{i=1}^{n} q_{i}\right\}=\left\{v_{1}, v_{2}, \cdots v_{m}\right\}$ is the atom set of $B$.

Let $A_{m \times m}^{B}$ is the matrix representation of $B$.

Let $G^{B}$ is the graph representation of $B$.

Let $v$ is the respondent's undergoing goal received by proponent.

(1)if $\left[\left(v \notin V^{B}\right) \cap\left(\neg V \notin V^{B}\right)\right]$ which indicates that there are no relevant information according to the proponent's knowledge, no recommendation plan is made.

(2)if $\exists v^{\prime} \in V^{B}, v^{\prime} \neq v, A_{m \times m}^{B}\left[v^{\prime}, v\right]=1$, then let $R=\left\{v^{\prime} \mid A_{m \times m}^{B}\left[v^{\prime}, v\right]=1, v^{\prime} \neq v\right\} \quad$ select $\quad v^{\prime} \in R \quad$ and recommend $v^{\prime}$ to the opponent as the alternative plan.

Negotiation Engine, with the support of Reason Engine, executes the selected negotiation plan and manages the negotiation process. Negotiation Engine serves as the communication interface where all information comes in and out. In more detail, Negotiation Engine evaluates the proposal from his respondent and generates his counter proposal. Once the negotiation process goes into stalemate and no concession or little concession is made, check if the "AskMore" condition holds to decide whether to continue the negotiation or enter "AskMore". After entering "AskMore" strategy, the following recommendation alternative plan process gets involved.
The belief base can be seen as the negotiation context [8] in which the goal is hold and the proposals are made. The utility is introduced as a measure of a proposal. It can be defined as a weighted sum of individual attribute evaluation.

Let $i=\left\{i_{1}, i_{2}, \ldots i_{i} \ldots, i_{m-1}, i_{m}\right\}$ are the negotiable issues; one-dimensional matrix $x=\left(x_{1}, x_{2}, \ldots, x_{m}\right)$ is the proposal; $x_{i}$ denotes the chosen option for issue $i_{i} ; B$ is the negotiation context, $b_{i}$ is the concrete context related to issue $i_{i}, \cup_{i=1}^{n} b_{i} \subset B$.

The utility can be represented as follows:

$U(B, x)=\sum_{i=1}^{n} w_{i} u_{i}\left(b_{i}, x_{i}\right)$

$u_{i}\left(b_{i}, x_{i}\right)$ is the utility of the chosen value $x_{i}$ for issue $i_{i}$ for the negotiator in the context $b_{i}$.

$w_{i}$ is the weight of issue $i_{i}, \sum_{i=1}^{n} w_{i}=1$.

Whether to accept the proposal or not is determined by following function.

$$
\operatorname{Accept}(B, x)=\left\{\begin{array}{l}
y e s, \text { if }\left(\forall x \in X, U(B, x) \geq U\left(B, x^{\prime}\right),\right. \\
\left.\quad \text { or } x \in X^{\prime \prime}\right) \\
n o, \text { else }
\end{array}\right.
$$

$x^{\prime}$ is the proposal for next time to send to the respondent.

$X$ represents all the possible set of proposals.

$X^{\prime \prime}$ represents all the proposed proposals.

Whether to ask the respondent for underlying goal or not is determined by following function.

$$
\text { Askmore }(B, x)=\left\{\begin{array}{l}
\text { yes, if }\left|m=\sum\left\{U\left(B, x^{i}\right)-U\left(B, x^{i-1}\right) \leq \delta\right\}\right|>\theta \\
\text { no and continue original negotiation , otherwise }
\end{array}\right.
$$

$x^{i}$ and $x^{i-1}$ represent the two successive proposals from the respondent.

$$
m=\sum\left\{U\left(B, x^{i}\right)-U\left(B, x^{i-1}\right) \leq \delta\right\} \text { represents the }
$$
times of the case in which the utility interval of the two successive proposals from the respondent is below the given utility value $\delta$.

$\theta$ represents the given threshold times, if $m$ exceeds $\theta$, then enter “AskMore” strategy.

\section{Example study}

We use an example to describe the negotiation process, focusing on the recommendation of the alternative plans to the respondent to solve conflicts in the negotiation and improve the negotiation efficiency. Notably, our model is not necessary to be restricted to recommendation as described above.

$\mathrm{X}$ is a company in the manufacturing industry. It has a goal to improve its competency in the global market. $\mathrm{X}$ believes that developing new products and 
increasing productivity are the steps to reach this goal. To increase productivity, $\mathrm{X}$ believes it needs to update product line hardware to extend working hours. The belief base of $X$ can be represented as: $B^{X}=\left\{b_{1}, b_{1} \rightarrow b_{2}, b_{1} \rightarrow b_{3}, b_{3} \rightarrow b_{4}, b_{4} \rightarrow b_{5}\right\}$,which is the initial negotiation context.

b1: improve competency; b2: develop new products; b3: increase productivity; b4: extend working hours; b5: update product line hardware

$\mathrm{Y}$ is a computer company specialized in $\mathrm{KM}$ (knowledge management) software. $\mathrm{Y}$ believes that encouraging organizational learning through KM software is an excellent way to improve the competency. Y is currently promoting the KM software. The belief base of $\mathrm{Y}$ can be represented as:

$$
\begin{aligned}
B^{Y}= & \left\{b_{1}, b_{1} \rightarrow b_{2}, b_{1} \rightarrow b_{3}, b_{1} \rightarrow b_{6}, b_{6} \rightarrow b_{7}, b_{4} \rightarrow \neg b_{3},\right. \\
& \left.b_{4} \rightarrow \neg b_{1}\right\}
\end{aligned}
$$

b6: encourage organizational learning; b7: adopt knowledge management systems software

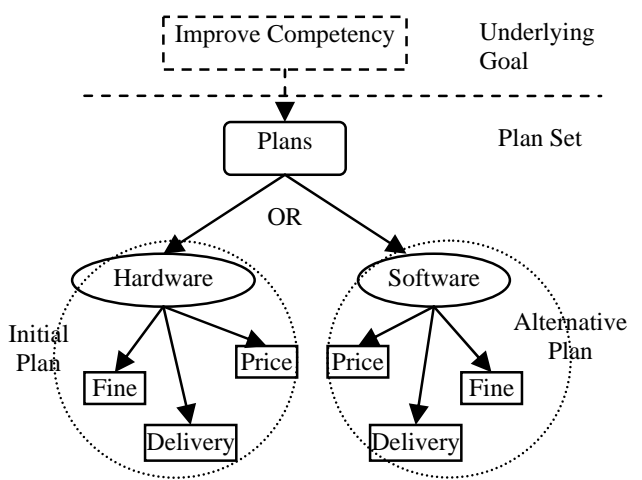

Fig.4: The underlying goal and the negotiation plan

At the beginning of the negotiation, $\mathrm{X}$ has his initial plan (buying the hardware from the company $\mathrm{Y}$ ) to achieve his goal. Agent $\mathrm{X}$ and Agent $\mathrm{Y}$ bargain through proposal and counter-proposal, respectively on behalf of company $\mathrm{X}$ and company $\mathrm{Y}$. Once little or no concession is made, Agent $\mathrm{Y}$ decides to ask Agent $\mathrm{X}$ for the underling goals, in order to improve the progress of negotiation. If informed by Agent $X$ of his underlying goal and the justifications for this goal ( $\left\{b_{1}, b_{1} \rightarrow b_{4} \rightarrow b_{5}\right\}$ ), Agent $\mathrm{Y}$ call Reason Engine to analyze the justification for the underling goal, and check whether the conflicts with his own belief exist or not.

After finding the conflicts $\left\{b \rightarrow b_{4} \rightarrow b_{5} \rightarrow b_{4} \rightarrow b_{5}\right\}$, Agent $\mathrm{Y}$ sends the conflicts to $\mathrm{X}$, as justification against the justification $\left\{b_{1} \rightarrow b_{4} \rightarrow b_{5}\right\}$, at the same time, selects the alternative plan $\left\{b_{1} \rightarrow b_{2} \rightarrow b_{6} \rightarrow b_{7}\right\}$ from his plan base, and initiates his preference about this plan and recommends it to Agent X, to tell Agent X that adopting knowledge management software can also improve the competency. Agent $\mathrm{X}$ adopts the alternative plan and renews his own belief base. After Agent $X$ 's renewing his belief, the new negotiation context $\mathrm{B}^{\prime \mathrm{X}}$ is shown in Table 2. Then, negotiation runs into another process to bargain about the alternative plan until success or fail. Certainly, if there are multiple alternative plans available, the next alternative plan can be recommended when current alternative plan comes to failure.

Let $O_{X \leftrightarrow Y}=(p, d, f)_{k} /\{\ldots\}$ is the proposal format, $(p, d, f)$ is the negotiation issues, $\{\ldots\}$ represent the justification, $k \in\{h, s\}, h$ is the hardware, $s$ is the KM software. The negotiation process illustrated in Table.3.

\begin{tabular}{|c|c|c|c|}
\hline \multirow{3}{*}{$\begin{array}{c}\text { Hardware } \\
\mathrm{B}^{\mathrm{X}}\end{array}$} & $\begin{array}{l}\text { Price(value } \\
\text { /utility) }\end{array}$ & $\begin{array}{r}\text { Delivery(value } \\
\text { /utility) }\end{array}$ & $\begin{array}{r}\text { Fine(value } \\
\text { /utility) }\end{array}$ \\
\hline & Weight:0.6 & Weight:0.3 & Weight:0.1 \\
\hline & \begin{tabular}{|l|l|l}
90 & 80 & 70 \\
$/ 0.28$ & $/ 0.30$ & $/ 0.3$ \\
\end{tabular} & \begin{tabular}{|l|l|l}
1 & 2 & 3 \\
0.14 & $/ 0.12$ & 0 \\
\end{tabular} & \begin{tabular}{l|l|l|}
$7 \%$ & $6 \%$ & $5 \%$ \\
0.08 & $0.04 / 0.02$ \\
\end{tabular} \\
\hline
\end{tabular}

Table 1: Initial context $\mathrm{B}^{\mathrm{X}}$ of $\mathrm{X}$.

\begin{tabular}{|c|c|c|c|}
\hline \multirow{3}{*}{$\begin{array}{c}\text { Software } \\
\mathrm{B}^{\mathrm{X}}\end{array}$} & $\begin{array}{l}\text { Price(value } \\
\text { /utility) }\end{array}$ & $\begin{array}{r}\text { Delivery(value } \\
\text { /utility) }\end{array}$ & $\begin{array}{r}\text { Fine(value } \\
\text { /utility) }\end{array}$ \\
\hline & Weignit & Weignt:0.1 & Weight:0.1 \\
\hline & \begin{tabular}{l|l|l}
30 & 30 & 25 \\
0.28 & $/ 0.30 / 0.3$
\end{tabular} & \begin{tabular}{l|l|l|} 
& 3 & 4 \\
0.14 & 0.12 & 0. \\
\end{tabular} & \begin{tabular}{|l|l|l|}
$8 \%$ & $7 \%$ & $6 \%$ \\
$/ 0.08$ & 0.04 & $/ 0.02$ \\
\end{tabular} \\
\hline
\end{tabular}

Table 2: Revised Context $\mathrm{B}^{\prime \mathrm{X}}$ of X.

\begin{tabular}{|c|c|c|}
\hline & Performtive & Content \\
\hline$X \rightarrow Y$ & Propose & $O_{X \rightarrow Y}=(70,1,7 \%)_{h} /\{\varnothing\}$ \\
\hline$X \leftarrow Y$ & Propose & $\ldots$ \\
\hline$\ldots$ & $\ldots$ & $\ldots$ \\
\hline$X \leftarrow Y$ & $\begin{array}{c}\text { Ask } \\
\text { (goals) }\end{array}$ & $O_{Y \rightarrow X}=(70,2,6 \%)_{h} /\{$ ?goal $\}$ \\
\hline$X \rightarrow Y$ & $\begin{array}{c}\text { Inform } \\
\text { (goals) }\end{array}$ & $O_{X \rightarrow Y}=(80,1,6 \%)_{h} /\left\{b_{1}, b_{1} \rightarrow b_{4} \rightarrow b_{5}\right\}$ \\
\hline$X \leftarrow Y$ & $\begin{array}{c}\text { Argue } \\
\text { (goals) }\end{array}$ & $O_{Y \rightarrow X}=(\varnothing)_{h} /\left\{b_{1} \rightarrow b_{4} \rightarrow b_{5} \rightarrow b_{4} \rightarrow b_{5}\right\}$ \\
$\wedge$ & $\ldots$ & $\ldots$ \\
\hline$\ldots$ & $\left.\ldots b_{5} \rightarrow b_{6}\right\}$ \\
\hline$X \leftarrow Y$ & $\begin{array}{c}\text { Recommend } \\
\text { (new plan) }\end{array}$ & $O_{Y \rightarrow X}=(\varnothing)_{s} /\left\{b_{1} \rightarrow b_{2} \rightarrow b_{6} \rightarrow b_{7}\right\}$ \\
\hline$X \rightarrow Y$ & $\begin{array}{c}\text { Adopt } \\
\text { (new plan) }\end{array}$ & $O_{X \rightarrow Y}=A c c e p t\left(\left\{b_{1} \rightarrow b_{2} \rightarrow b_{6} \rightarrow b_{7}\right\}\right)$ \\
\hline$X \rightarrow Y$ & Propose & $O_{X \rightarrow Y}=(70,2,6 \%)_{s} /\{\varnothing\}$ \\
\hline$X \leftarrow Y$ & Propose & $\ldots$ \\
\hline$X \rightarrow Y$ & Accept & $O_{Y \rightarrow X}=A c c e p t$ \\
\hline
\end{tabular}

Table 3: Excerpt of conversation of the whole negotiation process

\section{Related research}


In the multi-agent systems literature, various interaction and decision mechanisms for automated negotiation have been proposed and studied. Frameworks for automated negotiation have been studied [4]-[5], which usually assume that agents have complete, pre-set and fixed utilities and preferences, as well as complete awareness of the space of possible agreements (or deals). However, such strong conditions are often not satisfied. Agents may have limited, uncertain, or false information, preventing them from making optimal decisions about the fulfillment of their individual goals.

The traditional negotiation is Position Based Negotiation (PBN), in which, the involved parties firmly on their bargaining positions. They exchange proposals and counter-proposals in the anticipation that one or more parties will compromise to achieve a settlement of the dispute. One of the main limitations of this kind of negotiation framework is that they usually assume agents have complete and accurate information about the current world state and the results of actions. However in many practical situations, agents can't get complete and proper information, so the assumptions are not true and serious problems start to arise. In particular, bargaining could not be guaranteed to lead to agreements that truly maximize the participants' utilities [6].

Argumentation Based Negotiation (ABN) allows agents to exchange additional meta-information such as justifications, critiques, and other forms of persuasive locutions within their interactions, to argue over each others' beliefs, goals, and means to achieve these goals. It enables agents to gain a wider understanding of their counterparts, thereby makes it easier to resolve certain conflicts due to incomplete knowledge. It may be possible to reach agreements faster, and with a higher quality [7]-[8]. This approach has been advocated as a promising means to resolve conflicts within agent societies.

As a subset of Argumentation-Based Negotiation, Interest Based Negotiation (IBN) is a process that seeks to discover and satisfy the underlying interests of parties rather than to meet the stated positions or demands that they bring to negotiation. It is also known as win-win negotiation. Interests of a negotiation party tell others why he/she wants something. They reflect his/her underlying concern, needs or desire behind an issue. In interest based negotiation, the interests of participants are identified and explored, which helps them to understand the others' perspectives instead of simply reacting to the positions. By discussing the reasons behind the positions, mutually acceptable alternative plans to achieve this underlying goal. If the alternative plan is more attractive, the respondent may change his initial plan and preference and the negotiation process can avoid the conflicts other than solve the conflicts to make the negotiation successful. agreement is more likely to be reached. Interest based negotiation is therefore not only a much more powerful tool for conflict resolution, but also a good tool for coordination and cooperation. Tao et al[2] studied Interest Based Negotiation Automation, proposes a computational model for interest based negotiation automation which enables the automation of the fundamental elements of negotiation.

Pasquier et al[9] studied on the Interest Based Negotiation (IBN), presented a negotiation protocol that supports both alternate offers monotonic bargaining and given a reframing protocol. The "AskMore" protocol is similar to the protocol given in his paper, but our protocol mainly reasons on the underlying goal and we give a conceptual architecture for the protocol, at the same time, study the process how to recommend alternative plans basing on goals.

Rahwan et al[6] tried to equip intelligent agents with the ability to conduct interest based negotiation using argumentation based approach. They studied the relationships between agent's goals and the types of arguments that may influence other agents' decision, as well as defined a set of locutions that can be used in the negotiation procedure.

In reference[10], Rahwan et al Studied the relationships between argument-based negotiation and bargaining frameworks and demonstrated that if agents have false beliefs, then they may make decisions during negotiation that lead them to suboptimal deals. They described different ways in which argument-based communication can cause changes in an agent's beliefs and, consequently, its preferences over contracts and shown how the argumentation-based approach can improve both the likelihood and quality of deals.

\section{Conclusions}

During the negotiation, many conflicts or failure are due to the incomplete or incorrect knowledge about the negotiation goal. Negotiators (proponent \& respondent) can avoid this kind conflict through asking the underling goals of negotiation.

In this paper, we mainly focus on the underlying goal of negotiation and present Goal Directed Alternative Plan Recommendation Negotiation (GDAPLN), in which a negotiation agent can ask his respondent for the underlying goals of negotiation when the negotiation goes into stalemate. After getting the underlying goal of negotiation, the negotiation agent argues over the goals beliefs behind the goals and plans to achieve the goals, and recommend.

We give a conceptual model for GDAPLN and describe the functions of its components. Specially, consistency checking 、 conflict discovery 、 belief revisal and alternative plan recommendation is given. Finally, a case study is given to illustrate the negotiation process. 


\section{References}

[1] P. Bog, Towards Goals in Informed Agent Negotiation. AI 2006, LNAI 4304:423-432, 2006.

[2] X.H. Tao, Y. Miao and Z.Q. Shen, Interest Based Negotiation Automation. ICIC'2006, LNBI 4115:211-222, 2006.

[3] S.S. Fatima, M. Wooldridge and N.R. Jennings, An agenda-based framework for multi-issue negotiation. Journal of Artificial Intelligence, pp:152:1-45, 2004.

[4] R. Ashri, I. Rahwan and M. Luck, Architectures for negotiating agents. Lecture Notes in Artificial Intelligence vol. 2691: Multi-Agent Systems and Application III, pp:136-146, 2003.

[5] M.K. Cao, Y.Q. Feng and Y. Li, Negotiating Agent: Concept, Architecture and Communication Model. ICMLC’2005, LNAI 3930:53-63, 2005.

[6] I. Rahwan, L. Sonenberg and F. Dignum, Towards Interest Based Negotiation. AAMAS'03, July, 2003, Melbourne, Australian, pp:14-18, 2003.

[7] H.X. Huang, Z. Tan and J.W. Guo, Improved Model of Conventional Negotiation and Algorithm. Journal of Xi'An Jiao Tong University, 40(2):129-132, 2006.

[8] P. Yang, Y. Gao and Z.K. Chen, Persuasive Multi-Agent Multi-Issue Negotiation. Journal of Computer Research and Development, 43 (7):1149-1154, 2006.

[9] P. Pasquier, F. Dignum and I. Rahwan, Interest-Based Negotiation as an Extension of Monotonic Bargaining in 3APL. PRIMA'2006, LNAI 4088:327-338, 2006.

[10] I. Rahwan, L. Sonenberg and P. McBurney, Bargaining and Argument-Based Negotiation Some Preliminary Comparisons. ArgMAS'2004, LNAI 3366:176-191, 2004. 